This document is the accepted manuscript version of the following article:

Bula, C., Belosi, M. F., Eichin, M., Hrbacek, J., \& Meer, D. (2019). Dynamic beam current control for improved dose accuracy in PBS proton therapy. Physics in Medicine and Biology, 64(17), 175003 (14 pp.).

https://doi.org/10.1088/1361-6560/ab3317

\title{
Dynamic beam current control for improved dose accuracy in PBS proton therapy
}

\author{
C Bula, M F Belosi, M Eichin, J Hrbacek and D Meer \\ Center for Proton Therapy, Paul Scherrer Institut, 5232 Villigen, Switzerland \\ E-mail: christian.bula@psi.ch
}

\begin{abstract}
The step-and-shoot method of pencil beam scanning delivers the dose on a threedimensional grid in the target volume, with one dimension defined by the proton energy. While the dose per pencil beam may vary substantially within an iso-energy layer, the beam current typically remains constant. In this static operation mode, the inherent latency of the beam switchoff mechanism results in a lower limit for the deliverable spot dose, which may prevent the application of some of the low-weighted spots prescribed by the treatment planning system. To overcome this limitation, we introduced dynamic beam current control at the PSI Gantry 2, an innovative new approach successfully commissioned and in clinical operation since fall 2017. The control system was enhanced with a direct link to the vertical deflector located at the centre of the cyclotron. This connection allows much faster beam current changes $(\sim 0.1 \mathrm{~ms})$ and hence opens up the possibility of dynamically reducing the current for individual low-dose spots. We demonstrate that with this new dynamic operation mode, all spots are delivered as planned without compromising treatment time. We show by two independent and complementary methods that the delivered dose distribution is improved.
\end{abstract}

Keywords: proton therapy, pencil beam scanning, delivery system, minimum MU constraint, dose accuracy, dynamic current control, cyclotron, vertical deflector

\section{Introduction}

Proton therapy employs the Bragg peak, the characteristic dose deposition at the end of the proton range, to achieve improved dose conformity and reduced dose load on normal tissue compared to treatments with photons (Lomax 1999a, Ladra 2014). Early installations used passively scattered proton beams in combination with range modulators, collimators and compensators to shape the dose distribution (Koehler 1975, Koehler 1977). The majority of installations today use the technically more challenging pencil beam scanning (PBS) method developed at the Paul Scherrer Institute (PSI) (Pedroni 1995, Lin 2009). Among the advantages of PBS is the absence of patient-specific hardware and a higher flexibility in treatment modalities such as intensity modulated proton therapy (IMPT) (Lomax 1999b), allowing even better dose conformity proximal to the target volume and sparing of normal tissue (Cozzi 2001, Lomax 2003, Mock 2004, Arjomandy 2009).

The PBS method builds up the desired dose distribution by overlaying thousands of pencil beams with the Bragg peaks positioned on a three-dimensional grid in the target volume. Typically, two scanning magnets control the lateral beam position while the beam energy 
defines the range. In this configuration, the range is the slowest parameter to change, and therefore the dose spots are delivered as a series of layers perpendicular to the beam direction at constant energy. The beam current is typically adjusted in combination with the energy to compensate for variations in the beamline transmission, thus keeping the dose rate at the target approximately constant.

The step-and-shoot variant of PBS applies spots by setting the scanning magnets for the position of the next grid point and turning the beam on while keeping the lateral beam position constant. As soon as the desired spot dose is reached, the beam is turned off, and the magnets are adjusted for the next position. Due to the inherent latency of the beam switch-off procedure, a small amount of excess dose is delivered after sending the beam-off signal. This extra dose is proportional to the beam current and hence varies between energy layers. A correction for this extra dose is straight-forward, it can simply be subtracted from the nominal dose. Nevertheless, it sets a lower limit for the deliverable monitor units (MU), referred to as the minimum monitor unit (min-MU) constraint of the delivery system.

The weights of pencil beams are optimised by the treatment planning system (TPS) striving for adequate dose in the target volume and minimum dose in nearby healthy tissue. Depending on the clinical case, the optimisation is done either per field (single field uniform dose, SFUD) or, for anatomically and geometrically more challenging targets, simultaneously for all fields (IMPT), resulting in a particularly high modulation of spot weights within an energy layer.

In many planning systems, the min-MU constraint is either an integral part of the spot weight optimisation (Cao 2013, Shan 2018) or is met by an additional post-processing step. In the latter case, pencil beams with a dose below the lower limit can be handled in one of two ways: (1) the dose is rounded either down to zero or up to the min-MU value (Zhu 2010); (2) the dose is distributed over the neighbouring spots (Lin 2016).

The proton therapy facility PROSCAN at PSI (Schippers 2007a) consists of a superconducting cyclotron COMET and beamlines to the treatment areas: 3 gantries and a horizontal beam line for eye treatments. Gantry 1, the first PBS gantry in the world, was developed at PSI and started its clinical operation in 1996 (Pedroni 1995). It was used to treat patients until December 2018. Gantry 2, the 2nd generation scanning gantry developed at PSI (Pedroni 2004), is in clinical operation since 2013. Its design incorporates the experience gained from several years of operating Gantry 1 . The versatility of Gantry 2 allows its usage both for patient treatments and for technological developments. The third treatment room, Gantry 3, features a scanning gantry delivered by Varian Medical Systems (Koschik 2015). The integration of Gantry 3 in the PROSCAN environment was successfully completed in 2018.

During the initial phase of the Gantry 2 clinical operation, a combination of the abovementioned strategies for handling the min-MU constraint was employed. The PSI-developed TPS for Gantry 2 optimises the spot weights in terms of relative dose for a standard field dose. It supports a minimum spot weight constraint during the optimisation step by limiting the spot weights relative to the highest weighted spot in the field (SFUD) or plan (IMPT). When the plan is scaled to the prescribed dose, it may still contain spots that are below the hardware limitations of the delivery system. The therapy control system (TCS) takes over the post-processing step and decides on the fly whether a spot can be delivered or is skipped.

The min-MU limit may introduce significant distortions in the delivered dose distribution (Howard 2014, Kraan 2018). The effect can be mitigated on the beam delivery side by lowering the min-MU constraint. A common strategy adapts the beam current in combination with the energy such that the min-MU limit matches the lowest-weighted spot in the iso-energy layer, allowing all spots in the layer to be delivered as planned. However, because all spots in the layer are applied at the reduced beam current, this strategy results in an increased dose delivery time. 
In this publication, we report on a novel approach for achieving a lower min-MU constraint for the Gantry 2 delivery system, based on fast intensity modulation available in cyclotrons. The time required to change beam current was reduced by three orders of magnitude to $\sim 0.1 \mathrm{~ms}$ by enabling the TCS to directly control the vertical deflector at the centre of the cyclotron. At this time scale, temporary beam current adjustments for individual low-dose spots become feasible. We demonstrate with a sample of four clinical plans that this new dynamic operation mode:

- delivers all low-weighted pencil beams as planned

- has no effect on the field delivery time

- improves dose accuracy compared to the static operation mode

We consider dynamic beam current control to be an essential contribution to cyclotron-based PBS technology, especially in the context of new modalities such as rescanning and highintensity deliveries, where the number of low-weighted spots is high and thus the problem of min-MU constraints is more pronounced.

\section{Methods}

\subsection{Beam switch-off latency}

The procedure for applying a single proton pencil beam as it is implemented by the TCS of PSI's Gantry 2 is illustrated in figure 1. First, the TCS writes the dose requested for the spot to the preset register of the dose counter. Next, the dose delivery is started by switching the kicker magnet close to the exit of the cyclotron from the deflecting to the pass-through state such that the protons can travel in the beamline to the iso-centre. The dose reaching the target is registered by a planar ionisation chamber (IC) in the gantry nozzle. The readout electronics converts the ionisation current to a series of pulses representing monitor units (MUs). The calibration of the electronics is such that $1 \mathrm{MU}$ corresponds to 7250 protons at $150 \mathrm{MeV}$. The dose counter registers the pulses and issues the beam-off signal to the kicker magnet as soon as the MU count reaches the preset value. The kicker switches back to the deflecting state, preventing the protons from entering the beamline.

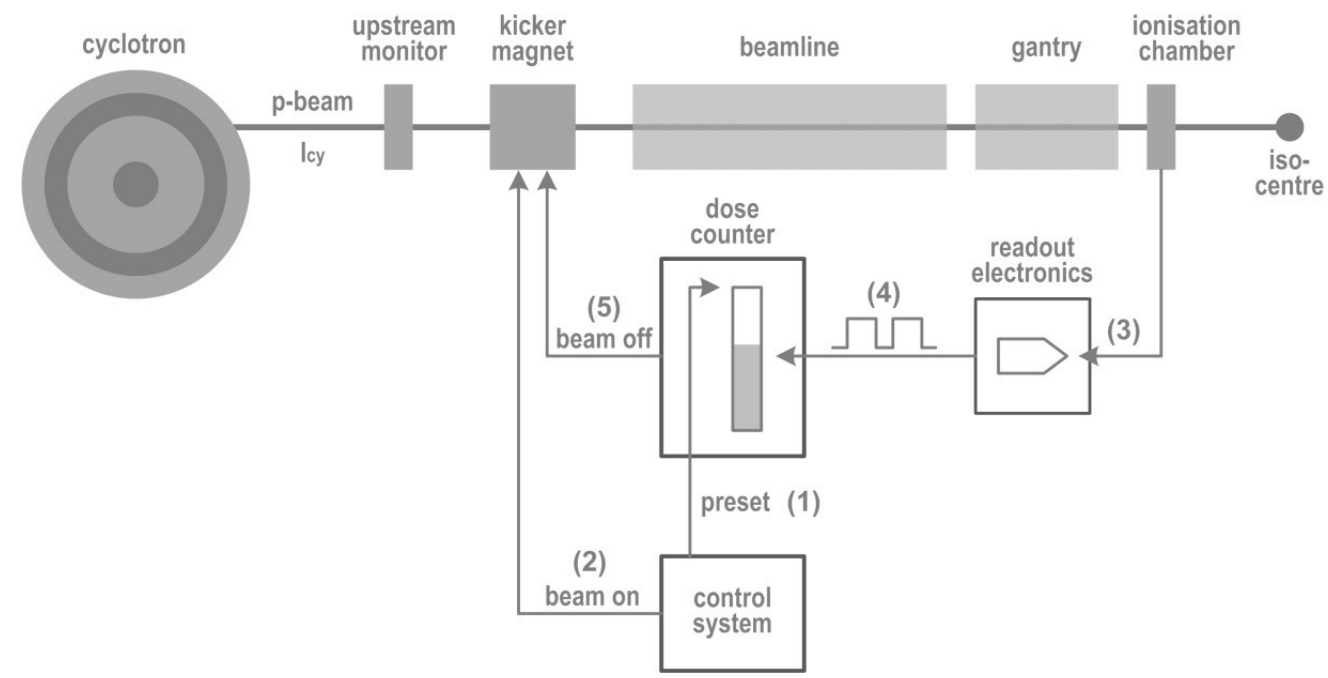

Figure 1. Procedure for proton pencil beam delivery: (1) set preset in dose counter; (2) switch kicker magnet to beam on; (3) digitise ionisation current; (4) register MU pulses; (5) switch kicker magnet to beam off.

The latency in the beam switch-off mechanism is approximately $230 \mu$ s, including contributions from the propagation of the beam-off signal from the control system to the kicker magnet ( 30 
$\mu \mathrm{s})$, the switching time of the kicker magnet $(100 \mu \mathrm{s})$ and the ion drift time in the IC (100 $\mu \mathrm{s})$. As a result, the ionisation current in the IC does not immediately drop to zero when the dose counter issues the beam-off signal, and therefore the readout electronics continues to generate MU pulses for a short time. Note that no protons reach the iso-centre after $130 \mu \mathrm{s}$, while the IC generates a current for another $100 \mu \mathrm{s}$. The resulting number of MUs registered in the dose counter in excess of the preset value is approximately 50 at the typical cyclotron current $\left(I_{c y}\right)$ of $200 \mathrm{nA}$ and dose rate of $250^{\prime} 000 \mathrm{MU} / \mathrm{sec}$ at iso-centre. The MU rate is proportional to $I_{c y}$ and depends on the beamline transmission as well as the energy-dependent ionisation density in the IC.

This switch-off latency can be corrected for by reducing the preset value with the expected excess counts. At Gantry 2, the correction is estimated for the first spot in the field from previously measured and tabulated values, stored as a function of beam energy and normalised to a current $I_{c y}^{\text {norm }}$ of $100 \mathrm{nA}$. For all remaining spots an exponentially weighted moving average of the measured excess counts in previous spots is used:

$$
<X>_{n}=\alpha \cdot X_{n}+(1-\alpha) \cdot<X>_{n-1}
$$

where $\langle X\rangle_{n}$ is the average excess count after $\mathrm{n}$ spots, $\alpha$ is the smoothing factor in the range [0..1] and $X_{n}$ is the excess count of the $n^{t h}$ spot obtained by comparing the number of registered MUs to the preset value. Note that both $\langle X\rangle_{n}$ and $X_{n}$ are normalized to $I_{c y}^{n o r m}$. For the smoothing factor $\alpha$, a value of 0.2 turned out to provide sufficient damping of fluctuations, while at the same time keeping the average $\langle X\rangle_{n}$ responsive to changes in external conditions. For a beam energy of $150 \mathrm{MeV}$, the normalized value $\langle X\rangle_{n}$ is approximately 25 MUs.

Figure 2 compares the estimated and measured excess counts for the first 100 spots in a clinical field. Only the first spot, with a correction based on tabulated values, shows an error of more than 5 MUs. The higher uncertainty for the first spot can be explained by day to day variations in the beamline transmission.

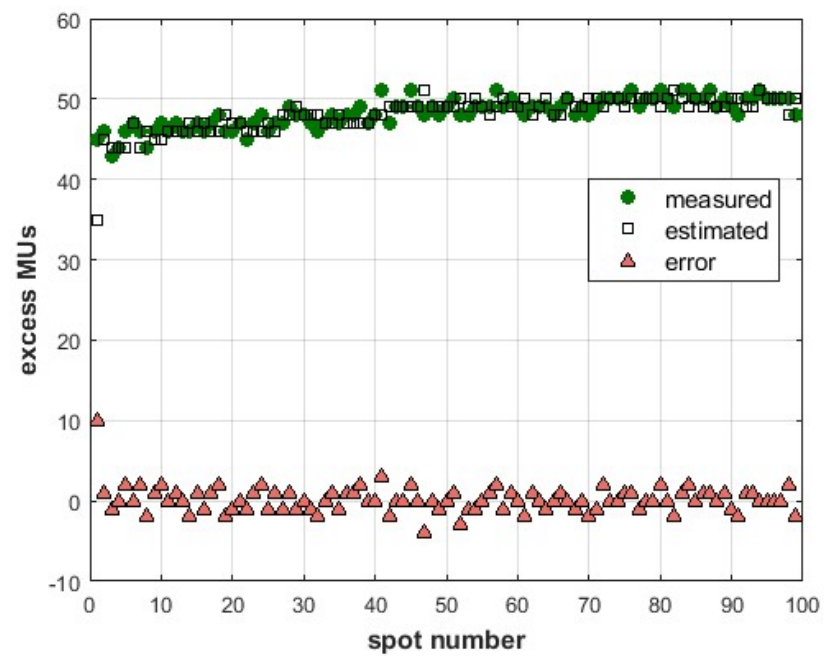

Figure 2. Estimated and measured excess MUs due to switch-off latency for the first 100 spots of a clinical field. The first spot, for which it is not possible to make an estimate based on previous measurements, shows a somewhat larger error.

\subsection{Minimum deliverable $\mathrm{MU}$}


The minimum deliverable MUs per proton pencil beam at Gantry 2 is given by the following two hardware limitations:

- The beam switch-off latency of $\sim 230 \mu$ s with the resulting excess dose. This effect is proportional to the upstream (or cyclotron) current $I_{c y}$, and therefore can be reduced by lowering $I_{c y}$. For the clinical operation at Gantry 2, a minimum current $I_{c y}^{\min }$ of $35 \mathrm{nA}$ is required, a limit enforced by a dose monitor at the exit of the cyclotron.

- The power supply of the kicker magnet requires a minimum delay between the beam-on (de-energise) and the beam-off (energise) signals. For simplicity reasons, this delay is enforced by a minimum preset $K$ of $5 \mathrm{MUs}$, independent of the upstream current.

Equation 2 summarises these two conditions as a lower limit for the deliverable MUs per pencil beam as a function of $I_{c y}$ :

$$
M U_{\text {low }}\left(I_{c y}\right)=<X>\cdot \frac{I_{c y}}{I_{c y}^{\text {norm }}}+K
$$

where $\langle X\rangle$ is the average excess dose normalized to $I_{c y}^{n o r m}$.

The standard current $I_{c y}^{s t d}$ for clinical operation at Gantry 2 is currently $200 \mathrm{nA}$ at a beam energy $E_{\text {beam }}$ of $150 \mathrm{MeV}$. For the other energies, the current $I_{c y}^{s t d}$ is adjusted to compensate the energy-dependent beamline transmission and thus achieve an approximately constant MU rate at the target.

To limit the variations in beamline transmission, we introduced an intensity compensation mechanism for the range of $100 \mathrm{MeV}$ to $200 \mathrm{MeV}$ : transmission changes due to the degrader are compensated by defocusing or focusing the beam with quadrupole magnets in front of a collimator (Rizzoglio 2018). Outside this range of $100 \mathrm{MeV}$ to $200 \mathrm{MeV}$, the transmission drops to $\sim 25 \%$ at $70 \mathrm{MeV}$ and rises to $400 \%$ at $230 \mathrm{MeV}$, relative to $150 \mathrm{MeV}$. This mechanism reduces the beam current adjustments necessary to achieve a constant MU rate to a factor of $\sim 16$, compared to $\sim 100$ without intensity compensation.

Based on equation 2, we can estimate the lowest deliverable number of MUs per pencil beam at the standard and the minimum beam current:

$$
\begin{gathered}
M U_{\text {low }}^{\text {std }}=M U_{\text {low }}\left(I_{c y}^{\text {std }}\right) \sim 55 \mathrm{MUs} \\
M U_{\text {low }}^{\text {min }}=M U_{\text {low }}\left(I_{c y}^{\text {min }}\right) \sim 14 \mathrm{MUs}
\end{gathered}
$$

Figure 3 compares the measured values of $M U_{\text {low }}^{\text {std }}$ and $M U_{\text {low }}^{\text {min }}$ as a function of $E_{\text {beam }}$. The results were obtained by applying a series of spots with decreasing dose up to the point where the TCS failed to deliver the spot, for energies ranging from $70 \mathrm{MeV}$ to $230 \mathrm{MeV}$ with $5 \mathrm{MeV}$ steps. Every data point is the average of five measurements.

The two data sets are representative for two operation modes of Gantry 2. In the static (conventional) mode, referred to as stMOD, the beam current is kept constant within an isoenergy layer, and the lower limit for the deliverable MUs is given by $M U_{l o w}^{\text {std }}$. The drop of $M U_{\text {low }}^{\text {std }}$ below $85 \mathrm{MeV}$ is a result of the (transmission-compensated) $I_{c y}^{s t d}$ reaching the maximum beam current of $\sim 450 \mathrm{nA}$ in the Gantry 2 setup (limited for safety reasons).

The new dynamic operation mode, labelled as dyMOD, supports beam current changes within an iso-energy layer and thus allows the delivery of spots with a weight as low as $M U_{l o w}^{\min }$. Note that the energy dependence of $M U_{\text {low }}^{\min }-K$ reflects the transmission of the beamline.

Spots with a weight in the range $\left[M U_{\text {low }}^{\text {min }}, M U_{\text {low }}^{\text {std }}\right]$ can be delivered in dyMOD only and demand a beam current adjustment for reducing the excess MUs. The adequate current for these spots is obtained from equation 2 as: 


$$
I_{c y}(M U)=\frac{(M U-K)}{<X>} \cdot I_{c y}^{n o r m}
$$

Note that the beam current $I_{c y}(M U)$ is to first order proportional to the spot weight in MUs, resulting in an almost constant beam-on time of $\sim 0.3 \mathrm{~ms}$ for low-weighted spots in the range $\left[M U_{\text {low }}^{\min }, M U_{\text {low }}^{\text {std }}\right]$.

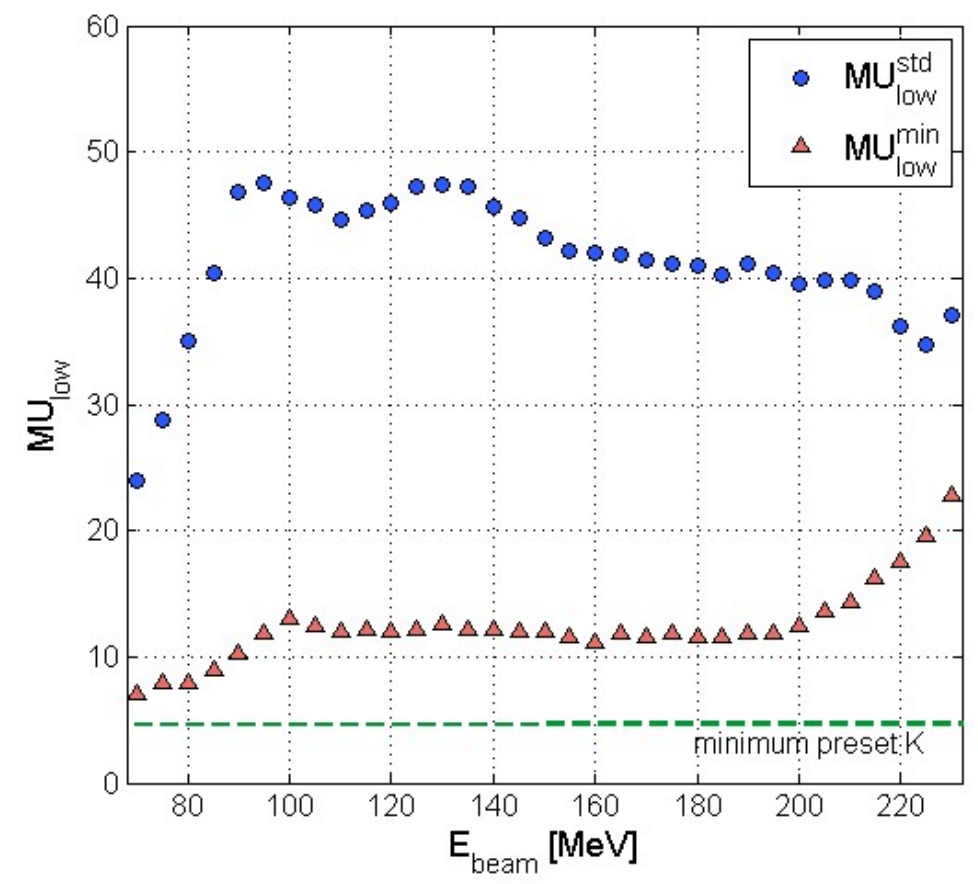

Figure 3. Minimum deliverable MUs at standard $\left(M U_{\text {low }}^{\text {std }}\right)$ and minimal $\left(M U_{\text {low }}^{\min }\right)$ beam current as a function of beam energy $E_{\text {beam }}$

\subsection{Beam current control}

The upstream current is regulated by the combination of a vertical deflector that consists of parallel plates located at the centre of the cyclotron, and a collimator. Protons on the innermost trajectories are deflected vertically in the electric field created by the high voltage applied to the deflector plates and are then partially blocked by the subsequent collimator. A zero voltage between the deflector plates results in maximum beam intensity while a voltage of $\sim 2.5 \mathrm{kV}$ suppresses the beam entirely. The power supply for the vertical deflector allows intensity variations at the exit of COMET within $50 \mu \mathrm{s}$. Control over the power supply is regulated by a dedicated access switch. 


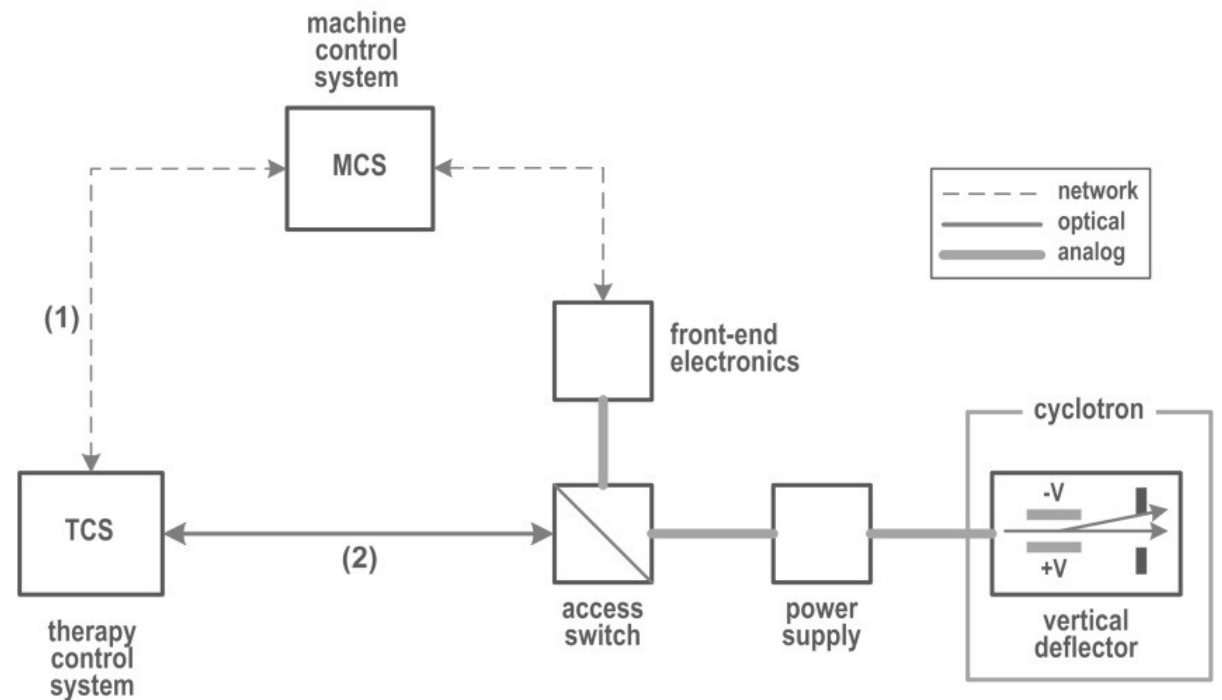

Figure 4. Beam current control of TCS via (1) MCS and front-end electronics in static operation mode and (2) via a direct optical link in dynamic operation mode. A deflector/collimator system at the centre of the cyclotron controls the beam intensity.

In stMOD, the machine control system (MCS) has exclusive access to the vertical deflector via the access switch. As illustrated in figure 4, MCS receives the beam current requests from TCS and implements a regulation loop running at $10 \mathrm{~Hz}$ to stabilise the current at the demand value. The network-based communication and the cycle time of the regulation loop realised in software result in a typical response time of $100 \mathrm{~ms}$ to a current change request.

In dyMOD the TCS communicates directly with the access switch via an optical link, allowing much faster response times of $\sim 100 \mu$ s for beam current changes. In this case, the conversion from beam current to deflector voltage is performed in the TCS. For that purpose, the TCS runs a calibration procedure at the beginning of each field, measuring the upstream current response as a function of the deflector voltage. During field delivery, the current is set according to the calibration (feed-forward) with no regulation mechanism in effect. As a safety measure, the beam current is sampled every $50 \mu$ s while delivering a spot and an interlock is raised if the measured current deviates by more than $30 \%$ from the nominal value.

\subsection{Vertical deflector calibration}

Controlling the vertical deflector directly from TCS requires a calibration that measures the upstream current as a function of the voltage on the deflector plates. The voltage is lowered from $2.5 \mathrm{kV}$ (complete suppression of beam) down to $0 \mathrm{~V}$ (maximum current) in steps of $\sim 40 \mathrm{~V}$. At each step, the current is sampled five times at $1 \mathrm{kHz}$ and averaged. The time required to run the calibration procedure is $\sim 0.7$ seconds.

In a second step, the TCS inverts the current vs voltage measurements to a voltage vs current lookup table (LUT) that provides the deflector voltage for the desired beam current. A precondition for the unambiguous inversion of the data is that the input data must provide monotonically decreasing current values for increasing deflector voltages. For this reason, an averaging filter is applied to the current values and data points that break the monotonically decreasing sequence are removed. 

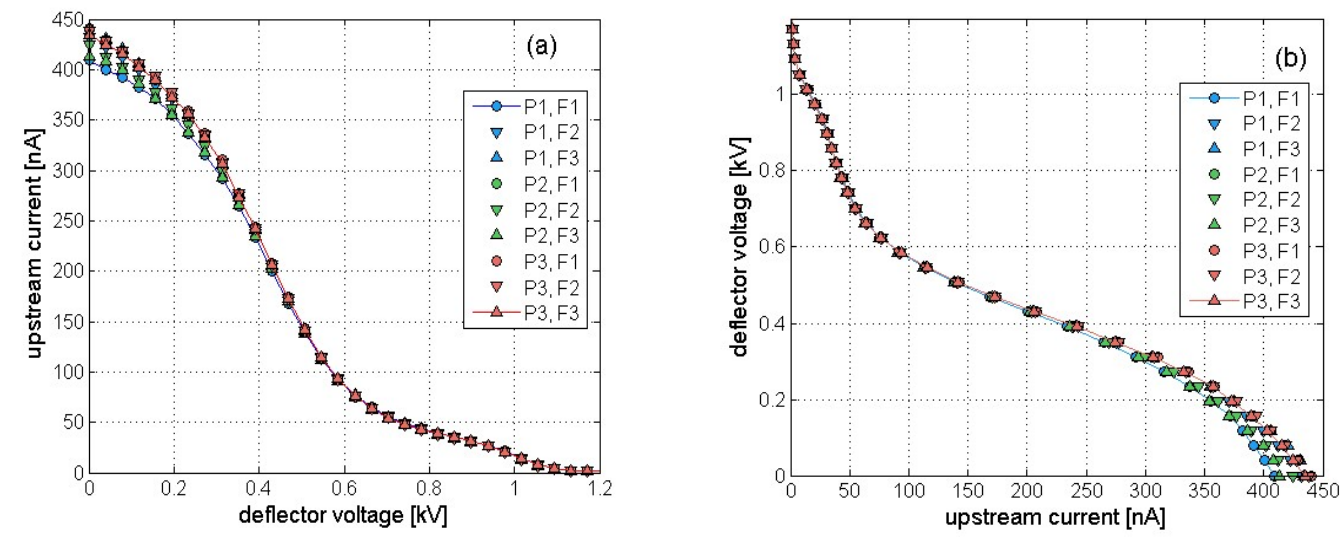

Figure 5. Vertical deflector calibration curves current vs voltage (a) and resulting lookup tables voltage vs current (b) measured at the start of 9 dose fields. All data were acquired within two hours.

Figure 5 shows the processed data and the resulting LUT for nine calibration runs. Although all data sets were collected within two hours, the measured current values at equal deflector voltage exhibit variations of up to $10 \%$. This considerable drift of the observed current response demands a calibration run at the start of each treatment field.

\subsection{Dose accuracy metrics}

To evaluate the effect of the Gantry 2 operation mode on the accuracy of the delivered dose distribution, four clinical plans (one IMPT and three SFUD) with a total of 12 clinical fields were delivered in Gantry 2 both in stMOD and dyMOD. For each field, the 2D dose profiles at a representative depth were measured in water by means of a 2D array of ICs (729 2D-array, PTW Freiburg $\mathrm{GmbH}$ ). The nominal dose distributions calculated by the TPS were compared to the delivered dose distributions with two independent and complementary methods.

The first three plans targeted immobilised tumours in the head and neck region with the dose applied in a single scan of the volume. The last plan treated a pediatric tumour in the abdomen region and made use of eight rescans to mitigate dose distortions effects caused by the respiratory motion.

The characteristics and location of the delivered plans are summarized in Table 1.

Table 1. Clinical plans analysed for dose accuracy comparison.

\begin{tabular}{|c|c|c|c|c|c|}
\hline Plan & Type & No of fields & $\begin{array}{c}\text { Dose per } \\
\text { fraction }[\mathrm{Gy}]\end{array}$ & No of spots & Location \\
\hline 1 & IMPT & 3 & 1.8 & 9154 & $\begin{array}{l}\text { head and neck } \\
\text { (1 scan) }\end{array}$ \\
\hline 2 & SFUD & 3 & 1.8 & 28836 & $\begin{array}{l}\text { head and neck } \\
\text { (1 scan) }\end{array}$ \\
\hline 3 & SFUD & 3 & 1.8 & 28078 & $\begin{array}{l}\text { head and neck } \\
\text { (1 scan) }\end{array}$ \\
\hline 4 & SFUD & 3 & 1.5 & 85839 & $\begin{array}{l}\text { abdomen region } \\
\quad(8 \text { rescans })\end{array}$ \\
\hline
\end{tabular}

\subsubsection{Dose reconstruction}


The TCS of Gantry 2 writes treatment-specific log files containing detailed information on each delivered pencil beam, such as beam energy, measured lateral position and delivered MUs. The analysis of these log files is an integral part of the patient-specific QA at PSI since the start of clinical operation in 2013.

The first method to evaluate the accuracy of dose delivery makes use of the Independent Dose Calculation (IDC) (Meier 2015), an in-house developed software package. The IDC uses the treatment logs to reconstruct the delivered 3D dose distribution on the patient anatomy. The analysis considers voxels with $10 \%$ or more of the prescription dose and calculates the relative difference $R D_{i}$ of reconstructed and nominal dose:

$$
R D_{i}=\frac{D_{I D C}^{i}-D_{T P S}^{i}}{D_{T P S}^{i}}
$$

where $D_{I D C}^{i}$ is the reconstructed and $D_{T P S}^{i}$ is the nominal dose in voxel $i$. Based on this information, the following metrics can be defined to quantify the agreement between the two dose distributions (Scandurra 2016, Belosi 2017):

- $R D^{\text {min }}: \quad$ lowest value of $R D_{i}$, i.e. maximum under-dosage

- $R D^{\max }: \quad$ highest value of $R D_{i}$, i.e. maximum over-dosage

- $\quad<R D>$ : mean value of all $R D_{i}$, i.e. average dose error

- $P R$ : pass rate, i.e. the percentage of voxels with $\left|R D_{i}\right|<1 \%$

\subsubsection{Dosimetric Measurement}

The second method recalculates the dose distribution in water and compares the resulting 2D profiles at a selected water-equivalent depth with the dosimetric measurement performed with the 2D array (Verisoft, PTW Freiburg) at the same depth in a water phantom (Lomax 2004). The two dose distributions are compared using a two-dimensional gamma analysis (Low 1998). The criteria for the gamma analysis were $2 \%$ maximum field dose difference and $2 \mathrm{~mm}$ distance to agreement. The resulting gamma score is used as the metric to quantify the agreement.

While the log file analysis compares reconstructed 3D distributions in patient geometry, this method is limited to comparing dose distributions in two dimensions in water at selected depths. On the other hand, this method is based on actual dose measurements.

\section{Experiments and results}

\subsection{Delivered pencil beams}

The capability of fast current changes, made possible by direct control of the vertical deflector of the cyclotron from the TCS, allows adjustment of the upstream current for individual lowdose spots to a level such that the spot becomes deliverable. This dynamic operation mode of Gantry 2 lowers the minimum deliverable MUs in a single spot by a factor of 3 to 4 compared to the static mode where the beam current is kept constant within an energy layer. Figure 3 compares the minimum spot dose as a function of the beam energy for the two modes.

Figure 6 illustrates the effect of the dyMOD on the delivery of a clinical field. While the planned spots below 60 MUs cannot be delivered (skipped) in stMOD (a), all spots can be delivered in dyMOD (c). Part (b) and (d) of the figure demonstrate that in stMOD the beam current remains constant within an energy layer while the dyMOD exhibits the characteristic beam current adjustments necessary to render the low-weighted spots deliverable.

The field selected for figure 6 (plan 1 / field 1 of table 2) is an extreme case with $11.8 \%$ of the pencil beams not delivered in the stMOD, representing $1 \%$ of the field dose. Even in this case, all spots were delivered as planned in dyMOD. On average over all cases, 3.6\% of the spots $(0.56 \%$ of MUs) could not be delivered in the stMOD. Conversely, for dyMOD, all spots could 
be delivered to all fields. Detailed information on all 12 clinical fields analysed is given in table 2.
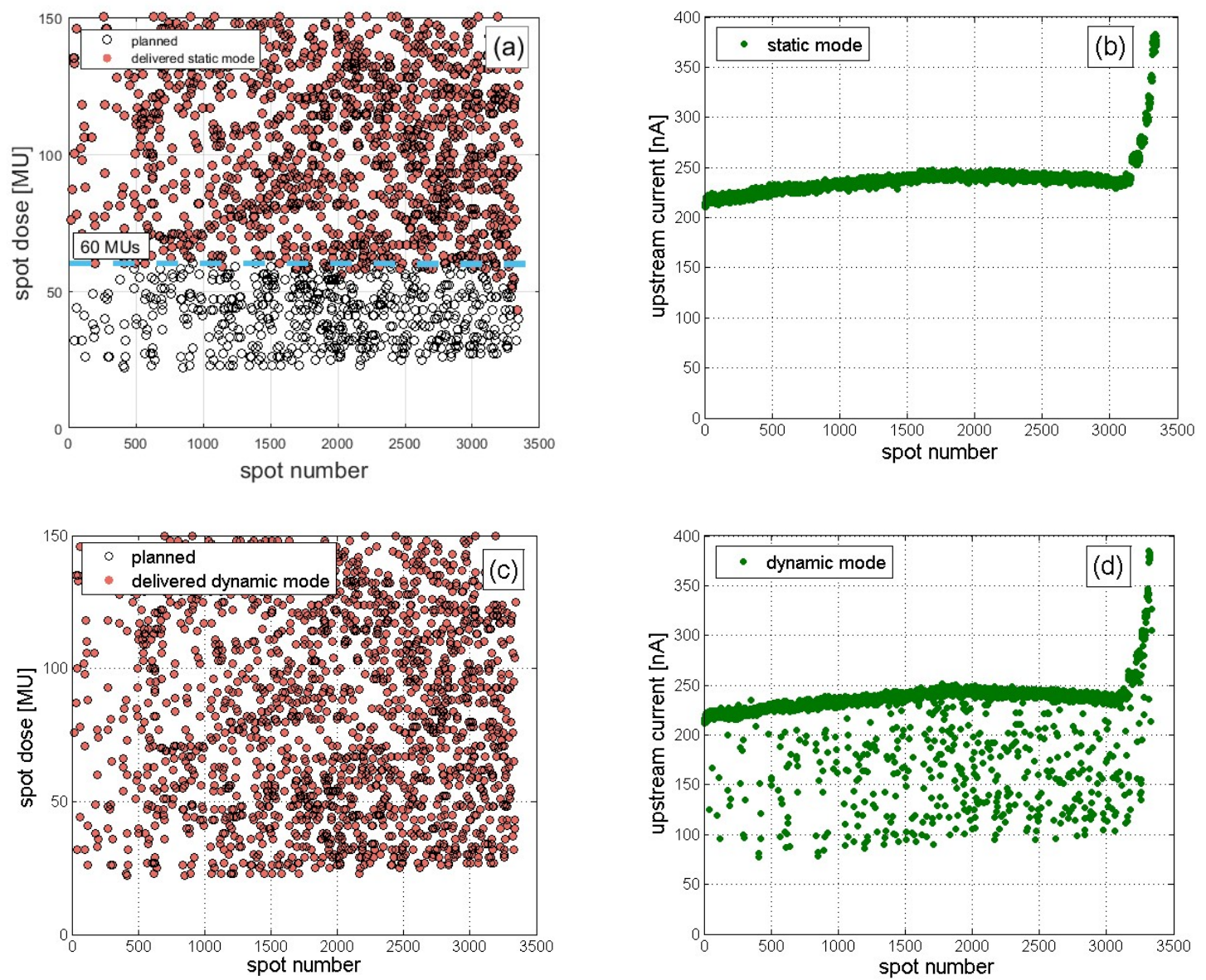

Figure 6. Delivery of a clinical treatment field in static $(a, b)$ and dynamic $(c, d)$ mode. Parts (a) and (c) show the weights of pencil beams planned and delivered while parts (b) and (d) depict the corresponding beam current.

Table 2. Number of skipped pencil beams, delivery times and results from the log file and gamma analysis for the four clinical plans. Results are presented per field and, where appropriate and available, per plan.

\begin{tabular}{|c|c|c|c|c|c|c|c|c|c|c|c|c|}
\hline \multirow[t]{2}{*}{$\begin{array}{l}\text { Plan/ } \\
\text { Field }\end{array}$} & \multirow[t]{2}{*}{$\begin{array}{c}\text { skipped } \\
\text { spots } \\
{[\%]}\end{array}$} & \multirow[t]{2}{*}{$\begin{array}{c}\text { skipped } \\
\text { MUs } \\
{[\%]}\end{array}$} & \multicolumn{2}{|c|}{$\begin{array}{c}\text { delivery } \\
\text { time } \\
\text { [s] }\end{array}$} & \multicolumn{2}{|c|}{$\begin{array}{c}R D^{\min } \\
{[\%]}\end{array}$} & \multicolumn{2}{|c|}{$\begin{array}{c}<R D> \\
{[\%]}\end{array}$} & \multicolumn{2}{|c|}{$\begin{array}{c}1 \% P R \\
{[\%]}\end{array}$} & \multicolumn{2}{|c|}{$\begin{array}{l}\text { gamma } \\
\text { score }\end{array}$} \\
\hline & & & stat & dyn & stat & $d y n$ & stat & dyn & stat & $d y n$ & stat & $d y n$ \\
\hline $1 / 1$ & 11.8 & 1.04 & 15.4 & 15.9 & -4.4 & -1.9 & -0.5 & 0.1 & 78.8 & 91.8 & 97.5 & 97.4 \\
\hline $1 / 2$ & 3.4 & 0.21 & 15.4 & 15.5 & -2.4 & -2.8 & -0.1 & 0.1 & 88.9 & 88.3 & 97.6 & 97.6 \\
\hline $1 / 3$ & 2.7 & 0 & 18.3 & 16.9 & -4.0 & -2.9 & -0 . & 0.1 & 80.7 & 90.5 & 100 & 100 \\
\hline $1 /$ all & 6.3 & 0.39 & 49.2 & 48.2 & -2.0 & -1.6 & -0.1 & 0.1 & 98.5 & 99.2 & - & - \\
\hline $2 / 1$ & 1 & 0.13 & 51.9 & 51.6 & 1.9 & -1.4 & -0.1 & 0.0 & 97.7 & 99 & 94.6 & 96.9 \\
\hline
\end{tabular}




\begin{tabular}{ccccccccccccc}
$2 / 2$ & 4.1 & 0.41 & 48.2 & 48.2 & -2.3 & -1.3 & -0.2 & 0.0 & 95.7 & 99.7 & 94.6 & 93.8 \\
$2 / 3$ & 5.7 & 0.52 & 37.2 & 37.6 & -3.5 & -3.3 & -0.3 & -0.1 & 78.7 & 80.9 & 91.5 & 92 \\
\hline $2 /$ all & 3.3 & 0.35 & 137.2 & 137.4 & -1.5 & -1.5 & -0.1 & 0.0 & 99.4 & 99.8 & - & - \\
\hline $3 / 1$ & 4.8 & 0.48 & 43.8 & 44.2 & -4.6 & -4.8 & -0.3 & -0.1 & 76.8 & 78.7 & 93.6 & 96 \\
$3 / 2$ & 3.6 & 0.33 & 46.5 & 46.7 & -4.8 & -3.4 & -0.2 & -0.1 & 74.5 & 81.4 & 99.2 & 100 \\
$3 / 3$ & 4.3 & 0.39 & 46.9 & 47.2 & -3.9 & -3.6 & -0.2 & 0 & 88.9 & 90.7 & 96.5 & 96.4 \\
$3 /$ all & 4.2 & 0.40 & 137.1 & 138.0 & -2.8 & -2.6 & -0.2 & 0.0 & 86.9 & 89.9 & - & - \\
\hline $4 / 1$ & 3.7 & 1.1 & 105.8 & 109.0 & -3.4 & -2.8 & -0.5 & 0.4 & 57.8 & 74.5 & - & - \\
$4 / 2$ & 3.6 & 1.25 & 111.8 & 114.5 & -2.9 & -3.0 & -0.6 & -0.3 & 66.6 & 83.6 & - & - \\
$4 / 3$ & 2.6 & 0.86 & 114.3 & 115.5 & -3.7 & -2.3 & -0.6 & 0.1 & 75.3 & 86.9 & - & - \\
$4 /$ all & 3.3 & 1.07 & 332.0 & 338.9 & -2.2 & -1.3 & -0.4 & 0.1 & 91.8 & 100 & - & - \\
\hline
\end{tabular}

\subsection{Field delivery time}

We investigated the influence of the operation mode on the field delivery time for the 12 clinical fields shown in Table 2. The analysis considered only the contribution of the spot application to the delivery time; beamline ramping at the start of the field and between rescans and layer switching time were excluded since these operations are carried out according to the same procedures in both modes. However, the analysis includes the beam-off time between spots, caused by reading out, analysing and logging of the detector values and setting the scanning magnets for the next spot position. The beam-off time amounts to $\sim 2.5 \mathrm{~ms}$ for spots delivered and $\sim 1.5 \mathrm{~ms}$ for spots skipped.

The results of these measurements are detailed in Table 2 and summarised in figure 7 . They demonstrate that the dyMOD has only a minor effect on the field delivery time. The average increase of delivery time amounted to $0.4 \%$ with a maximum value of $3 \%$. This is not surprising since, on average, only $4 \%$ of the planned spots $(0.6 \%$ MUs) were skipped and the additional delivery time for these spots in dyMOD of $\sim 1.3 \mathrm{~ms}(0.3 \mathrm{~ms}$ delivery and $1 \mathrm{~ms}$ added beam-off time) is moderate.

For two fields (Plan/Field 1/3 and 2/1) the delivery time in dyMOD was even slightly shorter than in stMOD. This can be explained by the time-dependent variations in the deflector characteristics as illustrated in figure 5. In the absence of a regulation mechanism for the dyMOD, the spots may be applied at a somewhat higher or lower beam current compared to the nominal value. As a result, the delivery time is somewhat shorter or longer compared to the stMOD where the regulation loop in the MCS stabilises the current at the nominal value. 


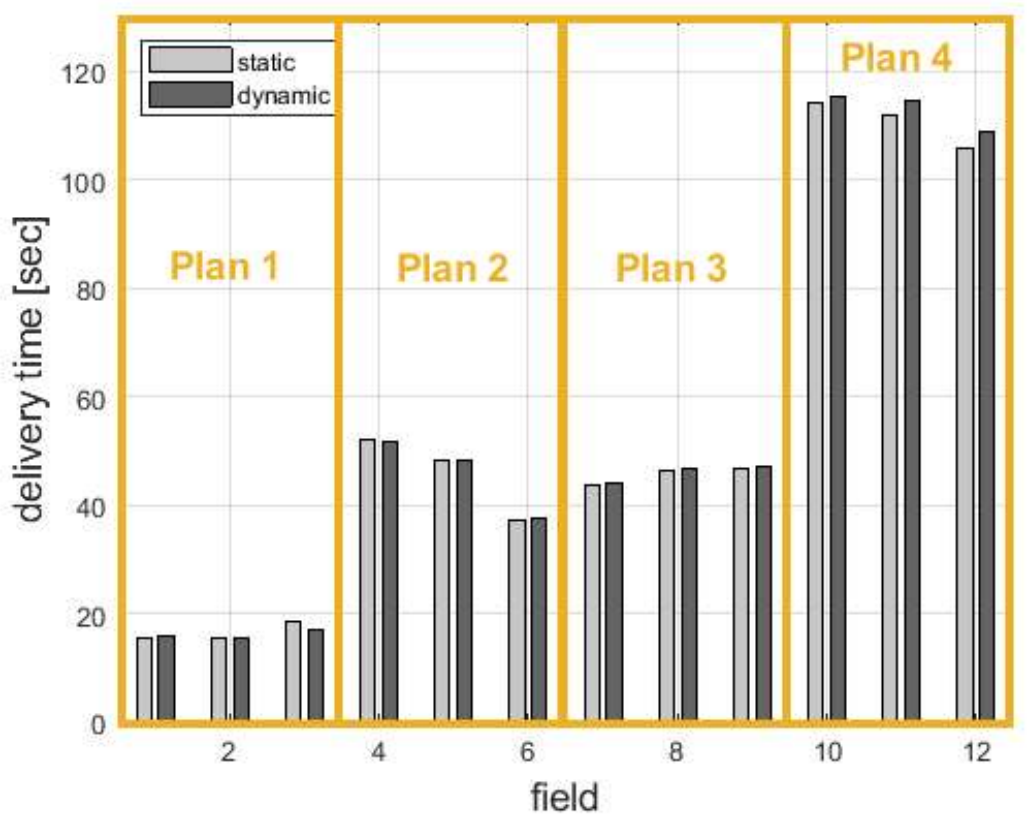

Figure 7. Delivery time of 12 clinical fields for static and dynamic operation mode. Only the contributions of spots are considered in the delivery time, while initial beamline ramping and energy changes are not included.

\subsection{Dose accuracy}

\subsubsection{Log file analysis}

The results of the comparison between nominal 3D dose distribution and the dose reconstructed in the patient anatomy based on the information in treatment log files are reported in table 2 for all 12 delivered fields. Figure 8 provides a visual representation of the data: to correlate the results with the amount of MUs not delivered in stMOD, the fields are grouped in three classes with a low $(<0.35 \%)$, medium $(0.35-0.70 \%)$ and high $(>0.70 \%)$ percentage of skipped MUs. Overall, the agreement between delivered and nominal dose is improved in dyMOD compared to the static case. As expected, the maximum under-dosage $R D^{\mathrm{min}}$ (a) is significantly reduced in dyMOD, while the maximum over-dosage $R D^{\max }$ (b) does not show a clear dependence on the delivery mode. Furthermore, the average dose error $\langle R D\rangle$ and the pass rate $P R$ exhibit a clear correlation to the proportion of skipped MUs in stMOD. 

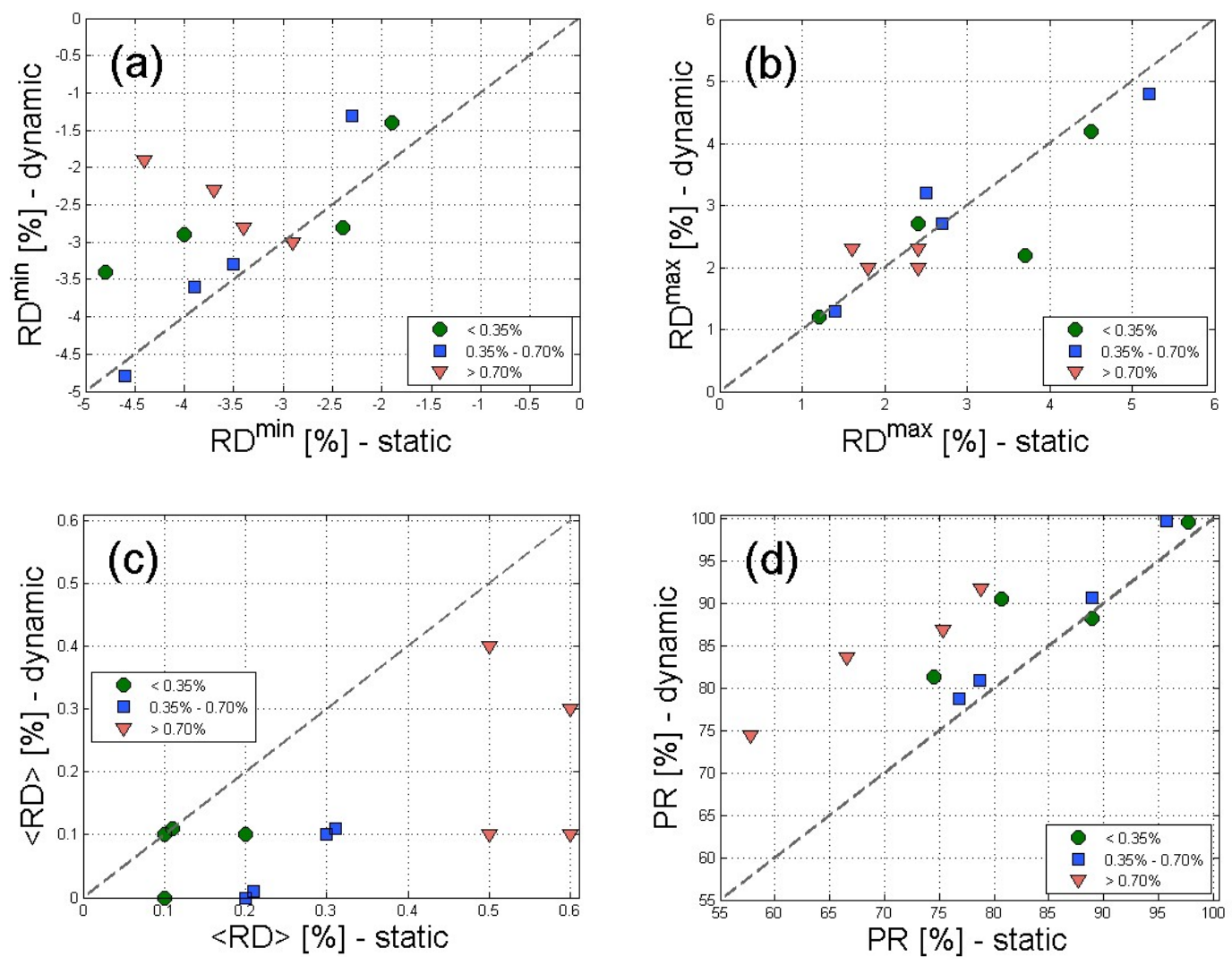

Figure 8. Results of log file analysis for the 12 delivered fields, grouped in three classes with small $(<0.35 \%)$, medium $(0.35-0.70 \%)$ and high $(>0.70 \%)$ percentage of skipped MUs when delivered in stMOD. The figures compare for stMOD and dyMOD the results for maximum under-dosage $R D^{\text {min }}$ (a), maximum over-dosage $R D^{\max }$ (b), absolute value of average dose error $\langle R D\rangle$ (c) and pass rate $P R(\mathrm{~d})$. The dashed line represents equal results for stMOD and dyMOD.

Figure 9 shows the relative difference between log file reconstructed dose and nominal dose per voxel. The difference is illustrated with the example of two fields (P1/F1 top row; P2/F2 bottom row) in a selected 2D slice and for the two modes (stMOD left-hand side, dyMOD right-hand side). For both fields, a clear under-dosage is visible for the stMOD delivery, which does not occur in the dyMOD. As expected, the under-dosage is located at the beam's entrance, typically the location of the least weighted pencil beams. 

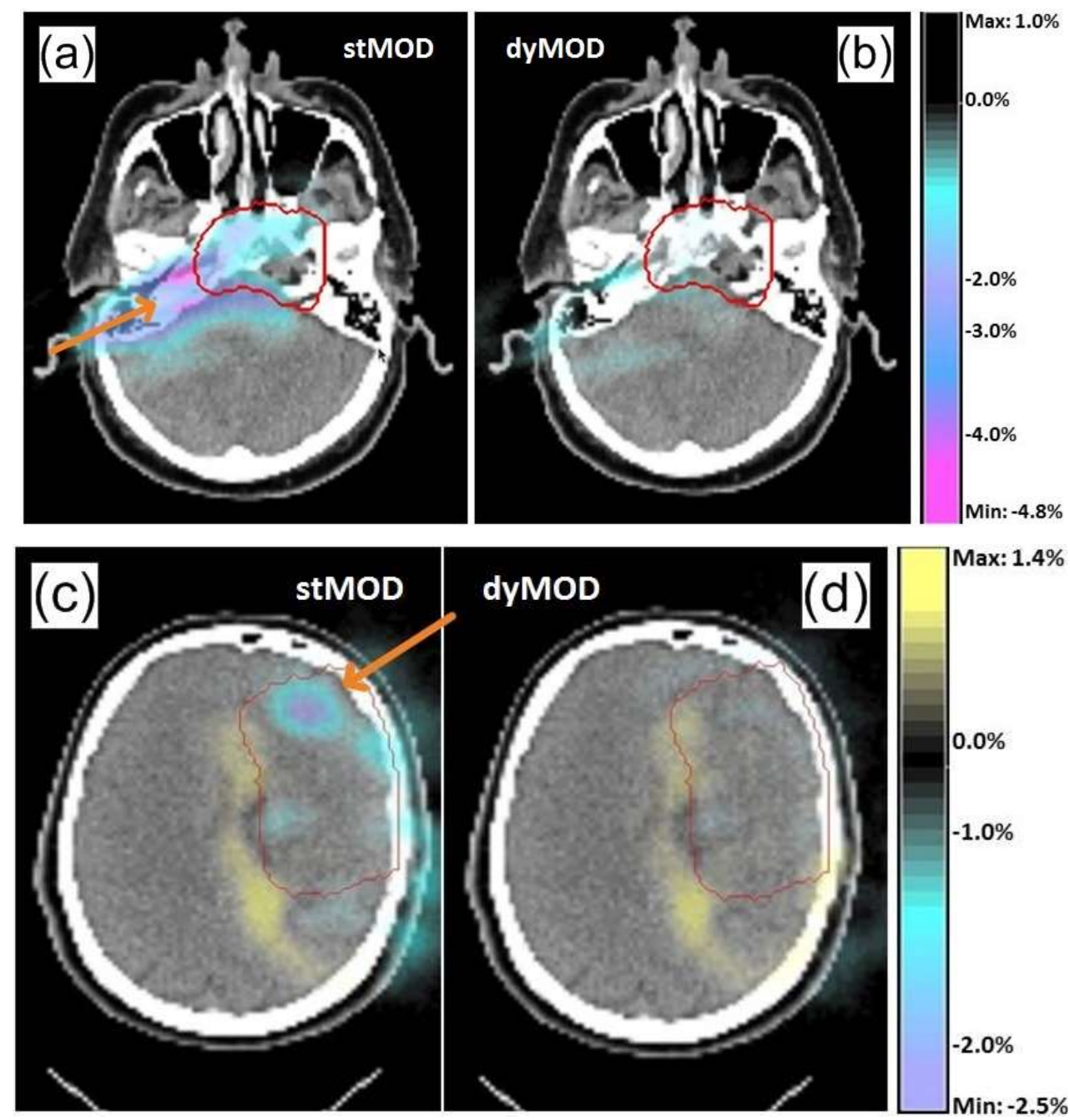

Figure 9. Relative difference $R D_{i}$ of delivered (IDC) and nominal (TPS) dose per voxel. The top row compares the results of an IMPT field (P1/F1) for stMOD (a) and dyMOD (b), while the bottom row compares stMOD (c) and dyMOD (d) for a SFUD field (P2/F2). The orange arrows indicate the field direction.

\subsubsection{Gamma analysis}

The dose distribution in water was measured at a specific depth with a $2 \mathrm{D}$ array of ICs and compared by a gamma analysis to the nominal distribution in water recalculated by the TPS. The results for three of the four plans are shown in figure 10. For most of the fields, the results are comparable, while two fields show a moderate improvement of the gamma score in dyMOD. In general, this method proved less sensitive than the log file analysis. 


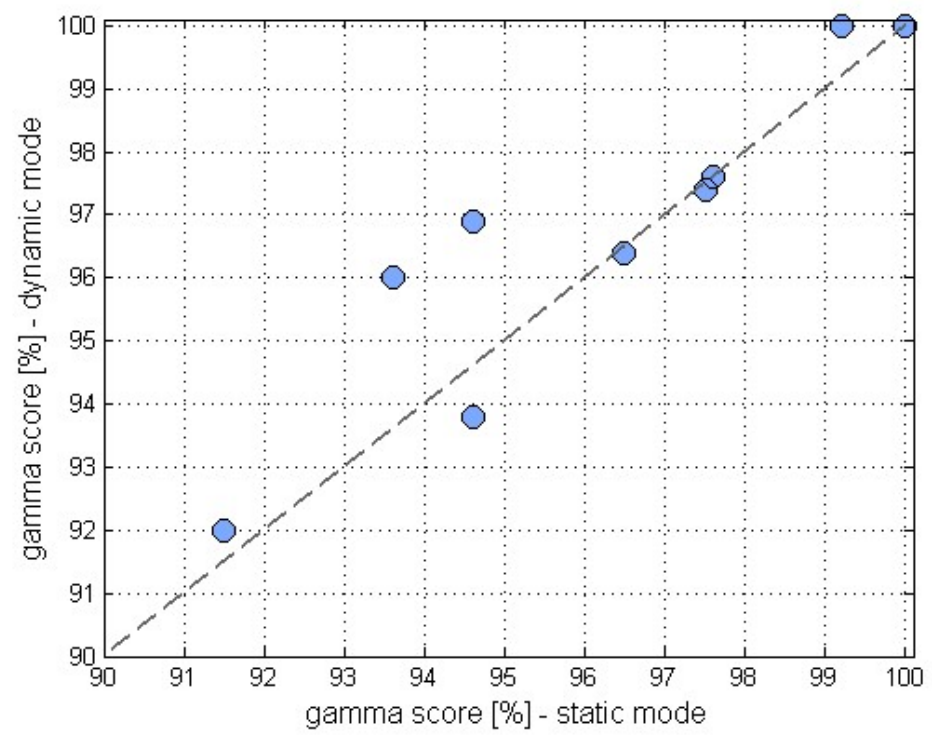

Figure 10. Gamma score of 2D dose distributions for nine fields. 


\section{Discussion and outlook}

In this paper, we report on a novel approach to lower the deliverable dose per pencil beam in our cyclotron-based delivery system for Gantry 2. By controlling the voltage of the vertical deflector system at the centre of the cyclotron via a direct optical link from the therapy control system (TCS), the beam current can be adjusted at a time scale of $\sim 0.1 \mathrm{~ms}$. This enables the TCS to reduce the current for individual low-dose pencil beams, thus lowering the min-MU constraint of our delivery system down to $\sim 10^{5}$ protons at $150 \mathrm{MeV}$. The system has been successfully commissioned and is in clinical operation since fall 2017.

Our motivation for enhancing the TCS by a dynamic beam current control in spot scanning was twofold. First, the direct link to the vertical deflector for fast current modulation was already implemented as part of the continuous line scanning project, a new delivery modality under development at PSI (Klimpki 2017). Second, our TPS implements a lower limit constraint on the relative weight of pencil beams compared to the highest weighted spots. This reduces the number of pencil beams below the absolute min-MU limit of the delivery system in the generated treatment plans, but cannot eliminate them completely. In the stMOD employed during the first years of operating Gantry 2, the beam current is adjusted in parallel with the beam energy to compensate for variations in the beam line transmission, with the goal of a constant dose rate at the iso-centre. However, no current adjustments are made for pencil beams below the min-MU limit, and as a consequence, these pencil beams are not delivered by the TCS (skipped spots). Dynamic beam current control was introduced to render these pencil beams deliverable and hence improve dose accuracy.

We evaluated the performance of this new dyMOD for spot scanning for a sample of four treatment plans (three SFUD and one IMPT) for a total of 12 fields. While deliveries in stMOD resulted in up to $12 \%$ of skipped spots (1.0\% of the field MUs), all spots were delivered as planned in dyMOD. We measured the minimum deliverable spot dose and demonstrated that the min-MU constraint for the delivery system is lowered in dyMOD by a factor of 3 to 4 compared to the stMOD. This limit depends on the minimum cyclotron current constraint for the clinical operation of the Gantry 2 beamline, currently set to $35 \mathrm{nA}$, namely half the nominal current for $230 \mathrm{MeV}$. By introducing an energy-dependent minimum cyclotron current, pencil beams with an even lower number of MUs could be delivered in dyMOD.

The field delivery time is only minimally affected by the operation mode. We found an average increase of the field duration in dyMOD of $0.4 \%$, with a maximum of $3 \%$ and even shorter durations for two fields. These fluctuations can be explained by the absence of a current regulation loop in dyMOD, resulting in higher or lower beam currents compared to the static mode. The extra time needed to dynamically adjust the current and deliver the low-dose spots which are skipped in the stMOD is negligible.

We analysed the accuracy of the dose delivery by reconstructing the $3 \mathrm{D}$ delivered dose distributions on the patient geometry using the information stored in the treatment log files and comparing it to the nominal distributions calculated by the TPS. By examining the relative voxel-by-voxel dose difference, we found that in general the agreement between delivered and nominal distributions is improved in the dyMOD. For all fields analysed, the dose error averaged over all voxels is lower, and the pass rate (percentage of voxels with less than $1 \%$ difference) is higher than or at least comparable to the results for the stMOD.

In a complementary analysis, we measured the dose profiles at selected depths with a 2D array of ionisation chambers and compared them to the predicted profiles in water by calculating the score of a $2 \mathrm{D}$ gamma analysis $(2 \%, 2 \mathrm{~mm})$. In contrast to the dose reconstruction from log files, this analysis is based on direct dose measurements, although in a $2 \mathrm{D}$ slice and in water. The results obtained with this complementary analysis confirm that the delivery in dynamic mode has the benefit of improving the accuracy of the delivered dose distributions as it allows the 
delivery of all planned MUs. However, the results are less conclusive than those obtained with the $\log$ file analysis.

The implementation of the dynamic operation mode required the following functional enhancements in the TCS:

- An on-line measurement of the average excess dose reaching the target after sending the beam-off signal, normalised to the cyclotron current. This enables the TCS to calculate the necessary reduction of the nominal current for delivering pencil beams with a dose below the min-MU limit.

- A calibration of the current at the exit of the cyclotron with respect to the vertical deflector voltage. This data is used to translate current requests to voltage settings at the deflector plate. We found that this calibration tends to drift considerably in time and needs to be updated frequently, i.e. before each field.

Although the system performs reliably, we realised that it would benefit from a regulation of the beam current during the field delivery. We encountered fluctuations of the current response to a deflector voltage of more than $20 \%$, even within the delivery time of a field. A regulation can be realised in various ways, e.g. by a hardware-based feedback loop comparing the measured to the nominal cyclotron current and acting on the deflector voltage. A similar system is already in place for the intensity modulation in continuous line scanning (Psoroulas 2017). A second option is a software-implemented correction of the beam current translated to the deflector voltage, based on the measured deviations of the actual current from the nominal value during the previous spots.

An alternative and more common approach to mitigate the min-MU limit of the delivery system adjusts the beam current to the lowest-weighted spot in the iso-energy layer. The time needed to change the current is in this case not critical because it can be done simultaneously with the adjustments in the beamline for the new energy. However, this procedure has the disadvantage that higher weighted spots in the layer are also delivered at the reduced beam current, resulting in a prolonged field delivery time. The size of this effect depends on many parameters specific to the treatment plan and the delivery hardware, e.g. the necessary beam current reductions and the total dose of the affected spots. We simulated this approach for the Gantry 2 system and the plans in this study, and found an average increase in field delivery time of $17 \%$ compared to the stMOD, with values ranging from $6 \%$ to $58 \%$.

Another possible source for a min-MU constraint of the machine hardware is the resolution of segmented ionisation chambers used for measuring the beam position. This constraint, however, does not apply for the Gantry2 system, where we have a redundant verification of the lateral position: the magnetic field of the scanning magnets is monitored with Hall sensors. This independent verification justifies widening the tolerances of the beam position check in the strip chamber for low-dose spots and eventually even dropping the check for the lowest-weighted spots $\left(<30 \mathrm{MUs}, \sim 2 \cdot 10^{5}\right.$ protons at $\left.150 \mathrm{MeV}\right)$.

In the context of new treatment modalities, the ability to deliver low-dose pencil beams will become even more important. The rescanning technique (Phillips 1992) aims at mitigating the adverse effects of respiratory motion on the dose distribution by irradiating the target volume multiple times and therefore reduces the dose per pencil beam by the rescanning multiplicity. In the breath-hold approach of motion mitigation (Gorgisyan 2017) the treatment time is crucial and high dose rates $(\sim 100 \mathrm{~Gy} / \mathrm{s})$ help to lower it. The corresponding increase in the excess dose results in more pencil beams requiring a temporary reduction of the beam current for delivery.

Whether the method described in this paper can be applied to a facility with a synchrotron depends strongly on the extraction method of the accelerator. The most promising method seems to be the RF-knockout method with which beam intensity can be modulated on the $10 \mathrm{~ms}$ time scale (Sato 2007). With this technique, the beam current of a synchrotron with slow 
extraction can be dynamically adjusted to improve the accuracy of the dose delivery. However, the noise on the beam current (spill ripple) of a synchrotron is usually much higher than that of a cyclotron, which will suppress the desired effect somewhat.

\section{Conclusion}

In this work, we present a novel method for lowering the minimum deliverable dose per proton pencil beam for the cyclotron-based delivery system of Gantry 2 at PSI. A direct link from the control system to the vertical deflector of the cyclotron lowers the time to change the beam current to $0.1 \mathrm{~ms}$, opening the possibility to reduce the beam current for individual low-dose spots. We demonstrated that low-weighted spots, which are not delivered in the standard operation mode, can now be applied as planned without an adverse effect on the field delivery time. By means of two independent methods we showed that the delivered dose distributions are improved with respect to the nominal distributions of the TPS.

\section{Acknowledgements}

The authors thank the technical staff of CPT in particular and PSI in general for the excellent installation and maintenance of the Gantry 2 delivery system. A special thank goes to Dr. Eros Pedroni for the conception and design of Gantry 2 and the continued inspiration. Finally, we would like to thank Dr. Zema Chowdhuri and Prof. Damien C. Weber for carefully reading this manuscript.

The authors have no relevant conflict of interest to disclose.

\section{References}

Arjomandy B, Sahoo N, Cox J, Lee A and Gillin M 2009 Comparison of surface doses from spot scanning and passively scattered proton therapy beams Phys. Med. Biol. 54 295-302

Belosi M F, van der Meer R, Garcia de Acilu Laa P, Bolsi A, Weber D C and Lomax A J 2017 Treatment $\log$ files as a tool to identify treatment plan sensitivity to inaccuracies in scanned proton beam delivery Radiotherapy and Oncology 125 514-19

Cao W, Lim G, Li X, Li Y, Zhu X R and Zhang X 2013 Incorporating deliverable monitor unit constraints into spot intensity optimization in intensity-modulated proton therapy treatment planning Phys. Med. Biol. 58 5113-25

Cozzi L, Fogliata A, Lomax A and Bolsi A 2001 A treatment planning comparison of 3D conformal therapy, intensity modulated photon therapy and proton therapy for treatment of advanced head and neck tumours Radiotherapy and Oncology $61287-97$

Gorgisyan J, Munck af Rosenschold P, Perrin R, Persson G F, Josipovic M, Belosi MF, Engelholm S A, Weber D C and Lomax A J 2017 Feasibility of Pencil Beam Scanned Intensity Modulated Proton Therapy in Breath-hold for Locally Advanced Non-Small Cell Lung Cancer International Journal of Radiation Oncology Biology Physics 99 1121-28

Howard M, Beltran C, Mayo C S and Herman M G 2014 Effects of minimum monitor unit threshold on spot scanning proton plan quality Med. Phys. 41091703

Klimpki G, Psoroulas S, Bula C, Rechsteiner U, Eichin M, Weber D C, Lomax A and Meer D 2017 A beam monitoring and validation system for continuous line scanning in proton therapy Phys. Med. Biol. 62 6126-43

Koehler A M, Schneider R J and Sisterson J M 1975 Range modulators for protons and heavy ions Nucl. Instrum. Methods 131 437-40 
Koehler A M, Schneider R J and Sisterson J M 1977 Flattening of proton dose distributions for large-field radiotherapy Med. Phys. 4 297-301

Koschik A, Bula C, Duppich J, Gerbershagen A, Grossmann M, Schippers J M, Welte J 2015 Gantry 3: Further development of the PSI PROSCAN proton therapy facility Proceedings, $\sigma^{\text {th }}$ International Particle Accelerator Conference (IPAC 2015): Richmond, Virginia, USA, May 3-8, 2015

Kraan AC, Depauw N, Clasie B, Giunta M, Madden T and Kooy H M 2018 Effects of spot parameters in pencil beam scanning treatment planning Med. Phys. 45 60-73

Ladra M M et al 2014 A dosimetric comparison of proton and intensity modulated radiation therapy in pediatric rhabdomyosarcoma patients enrolled on a prospective phase II proton study Radiotherapy and Oncology 113 77-83

Lin S, Boehringer T, Coray A, Grossmann M and Pedroni E 2009 More than 10 years experience of beam monitoring with the Gantry 1 spot scanning proton therapy facility at PSI Med. Phys. 36, 5331-40

Lin Y, Kooy H, Craft D, Depauw N, Flanz J and Clasie B 2016 A Greedy reassignment algorithm for the PBS minimum monitor unit constraint Phys. Med. Biol. 61 4665-78

Lomax A J, Bortfeld T, Goitein G, Debus J, Dykstra C, Tercier P-A, Coucke P A, Mirimanoff R O 1999a A treatment planning inter-comparison of proton and intensity modulated photon radiotherapy Radiother. Oncol. 51 257-71

Lomax A J 1999b Intensity modulated methods for proton therapy Phys. Med. Biol. 44 185-205

Lomax A J, Goitein M and Adams J 2003 Intensity modulation in radiotherapy: photons versus protons in the paranasal sinus Radiotherapy and Oncology 66 11-18

Lomax A J et al 2004 Treatment planning and verification of proton therapy using spot scanning: Initial experiences Med. Phys. 31 3150-57

Low D A, Harms W B, Mutic S and Purdy J A 1998 A technique for the quantitative evaluation of dose distributions Med. Phys. 25 656-61

Meier G, Besson R, Nanz A, Safai S and Lomax A J 2015 Independent dose calculations for commissioning, quality assurance and dose reconstruction of PBS proton therapy Phys. Med. Biol. $602819-36$

Mock U, Georg D, Bogner J, Auberger T and Pötter R 2004 Treatment planning comparison of conventional, 3D conformal, and intensity-modulated photon (IMRT) and proton therapy for paranasal sinus carcinoma International Journal of Radiation Oncology Biology Physics 58 14754

Pedroni E et al 1995 The 200-MeV proton therapy project at the Paul Scherrer Institut: Conceptual design and practical realization Med. Phys. 22, 37-53

Pedroni E et al 2004 The PSI Gantry 2: A second generation proton scanning gantry Z. Med. Phys. 14 2534

Phillips M H, Pedroni E, Blattmann H, Boehringer T, Coray A and Scheib S 1992 Effects of respiratory motion on dose uniformity with a charged particle scanning method Phys. Med. Biol. 37 223-34

Psoroulas S, Carmona P F, Klimpki G, Bula C, Meer D and Weber D C 2017 Challenges in fast beam current control inside the cyclotron for fast beam delivery in proton therapy Proceedings of the $21^{\text {st }}$ Int. Conf. on Cyclotrons and Their Applications 126-29 
Rizzoglio V, Adelmann A, Baumgartner C, Meer D, Snuverink J and Talanov V 2018 On the accuracy of Monte Carlo based beam dynamics models for the degrader in proton therapy facilities $\mathrm{Nucl}$.

Instrum. Methods A 898 1-10

Sato S, Furukawa T and Noda K 2007 Dynamic intensity control system with RF-knockout slowextraction in the HIMAC synchrotron Nucl. Instrum. Methods A 574 226-31

Scandurra D, Albertini F, Van Der Meer R, Meier G, Weber D C, Bolsi A and Lomax A J 2016 Assessing the quality of proton PBS treatment delivery using machine log files: Comprehensive analysis of clinical treatments delivered at PSI Gantry 2 Phys. Med. Biol. 61 1171-81

Schippers J M, Dölling R, Duppich J, Goitein G, Jermann M, Mezger A, Pedroni E, Reist H W and Vrankovic V 2007a The SC cyclotron and beam lines of PSI's new protontherapy facility PROSCAN Nucl. Instrum. Methods B 261 773-76

Schippers M, Duppich J, Goitein G, Hug E, Jermann M, Mezger A, Pedroni E 2007b First year of operation of PSI's new SC cyclotron and beam lines for proton therapy Proceedings of the $18^{\text {th }}$ Int. Conf. on Cyclotrons and Their Applications 15-17

Shan J, An Y, Bues M, Schild S E and Liu W 2018 Robust optimization in IMPT using quadratic objective functions to account for the minimum MU constraint Med. Phys. 45 460-69

Zhu X R, Sahoo N, Zhang X, Robertson D, Li H, Choi S, Lee A K and Gillin M T 2010 Intensity modulated proton therapy treatment planning using single-field optimization: The impact of monitor unit constraints on plan quality Med. Phys. 37 1210-19 